\title{
Spatial Evolution of Nonresonant Instabilities in the Precursors of Young Supernova Remnant Shocks
}

\author{
J. Niemiec* \\ Instytut Fizyki Jagdrowej PAN, ul. Radzikowskiego 152, 31-342 Kraków, Poland \\ E-mail: Jacek.Niemiec@ifj.edu.pl
}

\section{Pohl}

Institute of Physics and Astronomy, University of Potsdam, Karl-Liebknecht-Strasse 24/25, 14476 Potsdam, Germany

DESY, Platanenallee 6, 15738 Zeuthen, Germany

E-mail: marpohl@uni-potsdam.de

\begin{abstract}
The nonresonant cosmic-ray-current-driven instability that operates in the precursors of shocks in young supernova remnants may be responsible for magnetic-field amplification, plasma heating, and hydrodynamical turbulence, all of which have impact on the shock properties and particleacceleration processes. The temporal and spatial development of the instability is investigated here with Particle-In-Cell (PIC) simulations. Earlier PIC simulations used computational boxes with periodic boundary conditions which do not account for mass conservation in decelerating flows. Our current study for the first time uses a more realistic setup with open boundaries that permit inflow of plasma on one side of the simulation box and outflow at the other end. We demonstrate magnetic-field amplification as expected on the grounds of our earlier results. The effects of backreaction on CRs that slow down the initial relative drift velocity, limit further growth of the turbulence and lead to its saturation are also re-confirmed. We discuss a spatiotemporal structure of the shock precursor, including the evolution of CR distribution and the details of the saturation processes.
\end{abstract}

The 34th International Cosmic Ray Conference,

30 July- 6 August, 2015

The Hague, The Netherlands

\footnotetext{
* Speaker.
} 


\section{Introduction}

Shell-type supernova remnants (SNR) are ideal laboratories for the study of particle acceleration at nonrelativistic collisionless shocks. The micro-physics governing the coupling of energetic charged particles, amplified turbulent magnetic field, and colliding plasma flows, determines how suprathermal particles can be injected into Fermi-type shock acceleration processes, what is the level of cosmic-ray feedback and hence the nonlinearity of the system.

The aim of this work is to study the nonlinear development and saturation processes of the nonresonant cosmic-ray-current-driven instability that may be responsible for magnetic-field amplification in the precursors of shocks in young SNRs. Earlier studies of this instability [1] suggested that small-scale turbulence builds up that at peak intensity may reach a magnetic-field amplitude a factor of 10 or 20 higher than that of the homogeneous interstellar field [2, 3, 4, 5]. In the nonlinear phase, density fluctuations are imposed in, and bulk momentum is transferred to, the quasi-thermal plasma. Although this saturation mechanism seems to be in agreement with a quasi-linear theory of the nonresonant instability [6,7], an argument of the mass non-conservation was raised that questioned the correctness of a description of the strongly nonlinear phase of the instability in the simulations that employed periodic boundary conditions [8].

Here we report the preliminary results of our recent Particle-In-Cell (PIC) simulations of the nonresonant Bell's instability. In contrast to earlier PIC simulations that utilized computational boxes with periodic boundaries, our current study uses a more realistic setup with open boundaries that permit inflow of plasma on one side of the simulation box and outflow at the other end. In this way both the temporal and the spatial development of the instability can be investigated. The numerical PIC model and described in Section 2 and results of our test simulations are presented in Section 3.

\section{Simulation Setup}

The calculations are performed in the cosmic-ray (CR) rest frame. In this frame the electron-ion plasma moves with respect to CRs with a nonrelativistic shock velocity $v_{s h}$. The electrons are split into two populations: one of an inflowing charge-balanced electron-ion beam and a very lowdensity "excess" population $\left(\delta N_{e}=N_{e}-N_{i}=N_{C R}, N_{C R} \ll N_{e}\right.$ ) balancing the charge of CRs and initially at rest with them.

The numerical parameter set is similar to that used in [4], to permit a comparison with our earlier work. It very well recovers all physical characteristics of the nonresonant instability. The electron skin depth $\lambda_{\mathrm{se}}=c / \omega_{\mathrm{pe}}=4 \Delta$, where $\Delta$ is the grid cell size, $c$ is the speed of light, $\omega_{\mathrm{pe}}=\sqrt{e^{2} N_{e} / \varepsilon_{0} m_{e}}$ the electron plasma frequency, and $m_{e}$ the electron mass. Cosmic-ray ions are represented by a population of isotropic and monoenergetic particles with Lorentz factor $\gamma_{C R}=50$. The density ratio between the ambient and CR ions is $N_{i} / N_{C R}=50$ and the assumed plasma magnetization, given as the ratio of the predicted growth rate of the most unstable mode to the nonrelativistic ion gyrofrequency, is $\gamma_{\max } / \Omega_{i}=0.4$. The relative drift between CRs and the ambient plasma with velocity $v_{s h}=0.4 c$ is set antiparallel to the uniform magnetic field in the $-x$-direction. The initial electron temperature $v_{e, t h}=0.01 c$ is the same for both the ambient and the "excess" electron populations, and ions are in thermal equilibrium with the electrons. Computational constraints 
force us to use a reduced ion-electron mass ratio $m_{i}=50 m_{e}$. Nevertheless, this choice allows us to clearly separate the plasma and turbulence spatio-temporal scales. Preliminary simulations presented here use $N_{p p c}=9$ particles per cell per species, and statistical weights $w_{g}=0.02$ are applied to CRs and excess electrons to match a desired density ratios.

This model permits a stable injection of a beam of fresh plasma at one end of the numerical box. Beam particles are removed at the other end of the box, where the mean electron and ion fluxes are nearly identical and small-scale electromagnetic impulses generated by local charge imbalances are effectively absorbed by the open boundary. CR particles are kept inside the box and reflected if they meet the open boundaries for fields and the ambient plasma. The incoming electron-ion plasma pushes the excess electrons to the right, leaving the much heavier CRs initially unaffected. The simulated system quickly adjusts itself to the new situation, producing a suitable return current, exactly as one would envision a real plasma to respond. The excess electrons concentrate at the right boundary. They cannot be simply removed because that would violate charge conservation. With the evolution of the nonresonant instability towards a nonlinear stage, density and field structures are produced which further focus the plasma into compact islands. However, on account of the high density ratio, the concentrations in excess electrons are a small perturbation to the total electron distribution and thus numerical artifacts at the boundary have a much smaller amplitude than the turbulence in the region of interest. Therefore, the plasma outflow boundary does not have a noticeable influence on the distribution of CR particles.

To fully evaluate the nonlinear development of Bell's instability in both space and time, we perform large-scale simulations using two-dimensional numerical grids in the $x-y$ plane with periodic boundary conditions applied in the transverse direction. The preliminary results presented here are based on two test simulations. The first one utilized numerical box with $L_{x} \times L_{y}=32,000 \times$ $2400 \Delta^{2} \approx 72 \times 5.4 \lambda_{\max }^{2}$, and followed the evolution for 10.2 linear growth times. The second test run used a long and narrow grid with $L_{x} \times L_{y}=80,000 \times 480 \Delta^{2} \approx 180 \times 1.1 \lambda_{\text {max }}^{2}$ that enabled us to reach $t=20 \gamma_{\max }^{-1}$, which is in the instability saturation phase. Full-scale numerical experiments are planned with transverse sizes of $L_{y} \sim 10 \lambda_{\max }$, grid lengths of $L_{x} \sim 120,000 \Delta$, and simulation times of at least 30 linear growth times, $t \gamma_{\max } \gtrsim 30$.

To stabilize the plasma against nonphysical effects on such long times scales we apply a numerical PIC model used recently in [9]. The model takes advantage of second-order particle shapes (TSC - Triangular-Shaped Cloud) and uses a second-order FDTD field-solver with a weak Friedman filter [10] to suppress small-scale noise. The simulations were performed with a modified version of the TRISTAN code [11], which was adapted to work in 2D3V and parallelized using MPI [2].

\section{Results}

Figure 1 shows the $x-y$ distribution of the magnetic-field component $B_{z}$ at the end of the first of the test simulations at $t=10.2 \gamma_{\max }^{-1}$. The temporal growth of the Bell's nonresonant modes can be traced through a spatial increase in the amplitude from the left to the right side of the numerical grid. The waves appearing at $x / \Delta \approx 8.5 \times 10^{3}$, after the electron-ion beam has propagated through the $\mathrm{CR}$ plasma for $t \approx 6 \gamma_{\max }^{-1}$, move with the plasma and have wave vectors parallel to the plasma drift with wavelength of $\sim \lambda_{\max }$. Their amplitude at $x / \Delta \approx 2.9 \times 10^{4}$ reaches $\left\langle\delta B / B_{\| 0}\right\rangle \sim 6$ (compare Figs. 


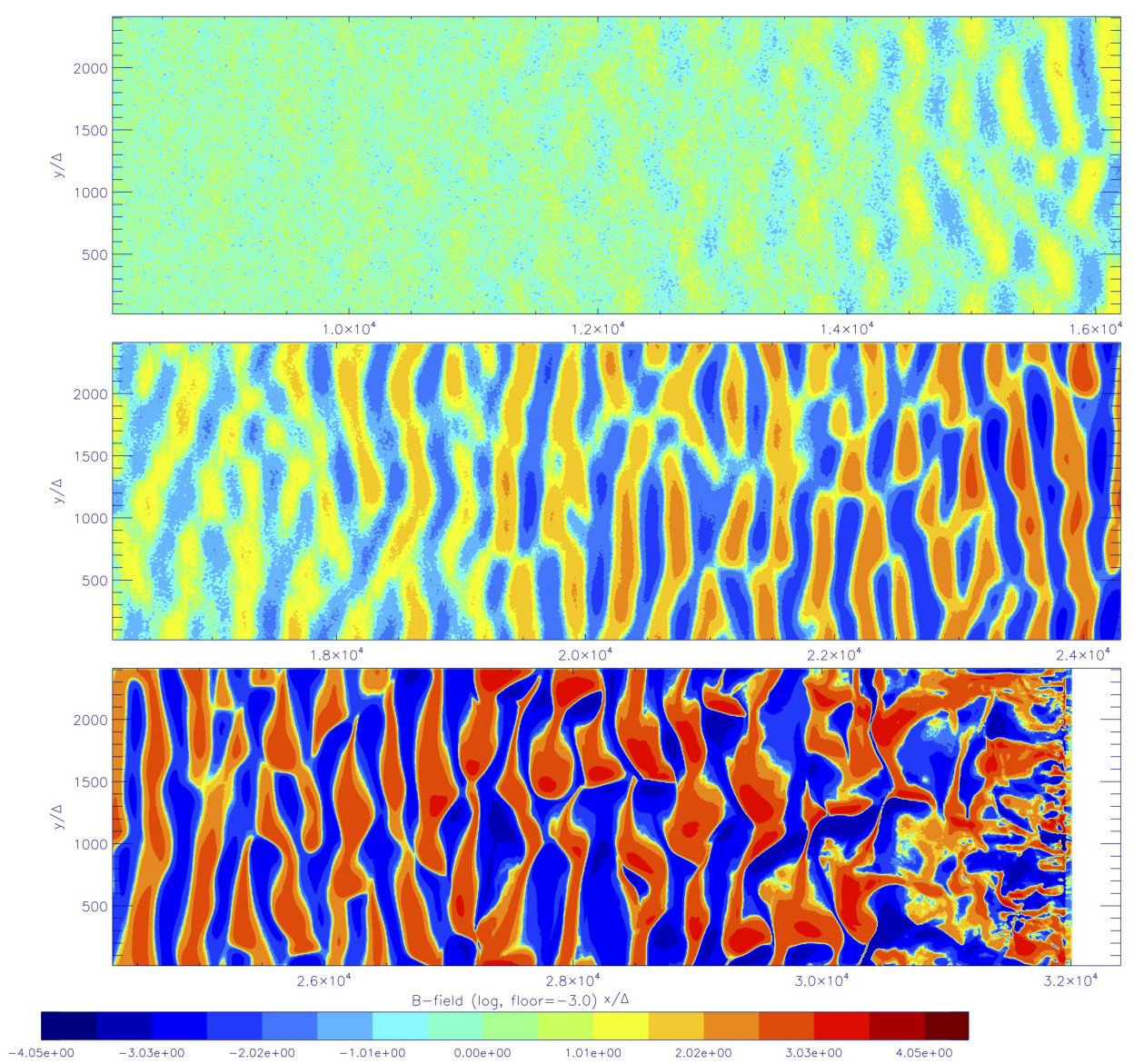

Figure 1: Distribution of the magnetic-field component $B_{z}$ in sign-preserving logarithmic scaling at the end of our smaller-box simulation at time $t=10.2 \gamma_{\max }^{-1}$. Drifts and the wavevector of the nonresonant instability are in the $x$-direction. The right boundary is at $x / \Delta \approx 3.2 \times 10^{4}$. Note that the boundary conditions for particles are applied 50 cells before the boundary for fields.

1 and 2 in [4]). By this point the magnetic turbulence structure deviates from a purely parallel mode and the dominant length scale starts to exceed $\lambda_{\max }$. Similar observations can be made for the motional electric field, shown as $E_{y}$ component in Figure 2. Turbulent structure in $E$ becomes apparent in the nonlinear stage, once $\delta B \gtrsim B_{\| 0}$.

The electromagnetic turbulence is accompanied in the nonlinear phase by density fluctuations in the quasi-thermal plasma. As the turbulent magnetic field becomes comparable in strength to the uniform field, the plasma parcels begin to move, collide, and concentrate into turbulently moving filaments, thus causing deviation from the purely-growing parallel mode. This is demonstrated in Figure 3, in which the ambient ion density is presented in the region where the magnetic field amplitude is highly nonlinear. Density and velocity fluctuations also lead to heating of the ambient plasma.

These characteristics of the nonresonant instability are similar to those observed in our earlier simulations employing periodic boundary conditions (see also $[5,8,3]$ ), but now both the temporal and the spatial development of the instability can be investigated. Note that numerical effects arising from imbalances at the open boundary are evidently confined to a thin layer of thickness 


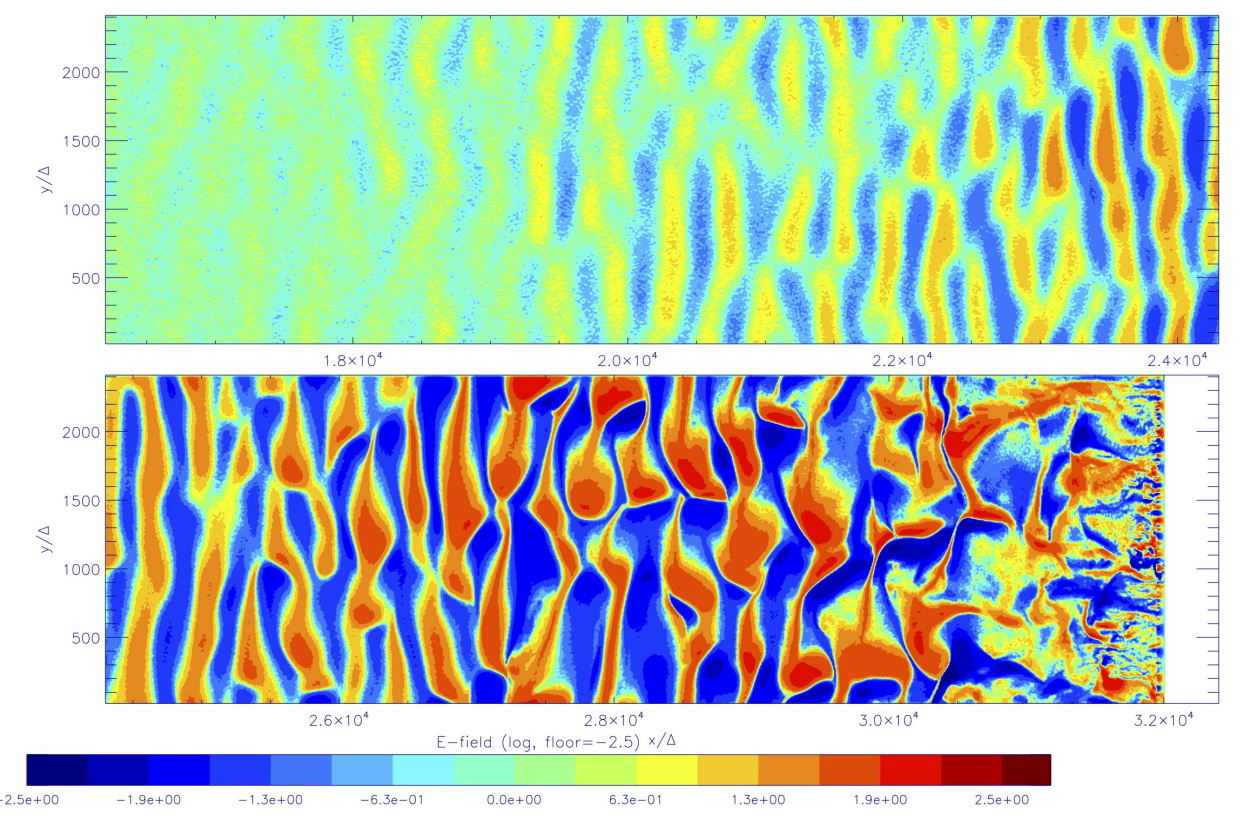

Figure 2: Distribution of the electric-field component $E_{y}$ in sign-preserving logarithmic scaling. Electric fields are associated with magnetic waves that move with the thermal plasma (see Fig. 1).

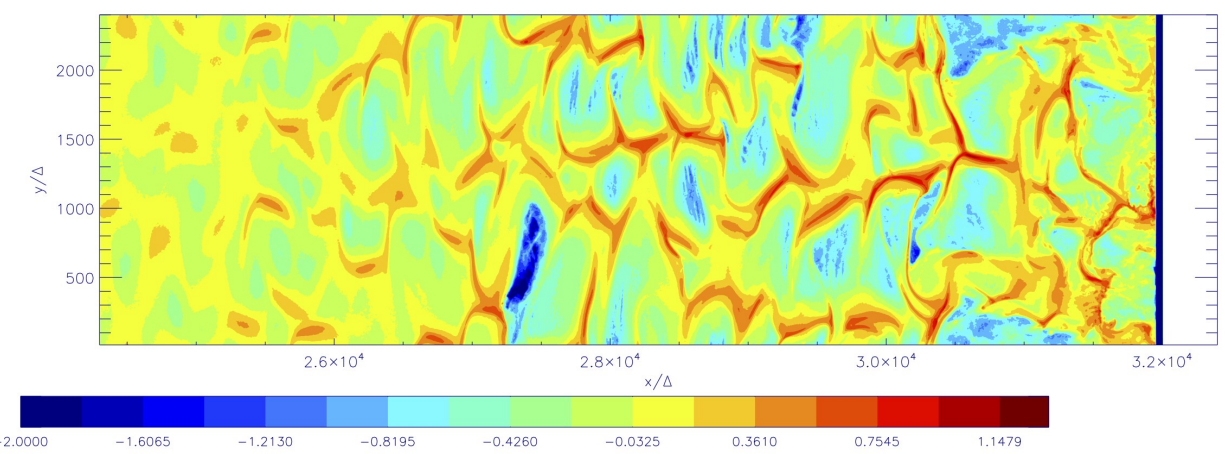

Figure 3: Density of ambient ions in a region of highly nonlinear magnetic turbulence near the right boundary of the numerical box. Logarithmic scaling is used. Note that particles of the electron-ion beam are removed 50 cells before the boundary for fields (see Fig. 1).

$\delta x / \Delta \lesssim 2 \times 10^{3}$. Numerical artifacts causing distortions in the electromagnetic turbulence and plasma density at this boundary layer have a much smaller amplitude than the turbulence in the physical region. This disturbed layer does not significantly grow when the system evolution is followed to a later times. In fact, as our second test run with a much longer simulation box reveals, the layer starts even to shrink due to the CR drift in the beginning of the strongly nonlinear phase (see below). Once the electron-ion beam slows down the unphysical region remains stable.

Further insight into the development of the nonresonant Bell's instability is given through results of our second test simulation performed on a long numerical grid with $L_{x}=80,000 \Delta$. Figure 4 shows the phase-space plot for the ambient plasma electrons and ions at the end of this run at $t=20 \gamma_{\max }^{-1}$. One can see that at locations $x / \Delta \gtrsim 35,000$, that correspond to a region of strong nonresonant turbulence, the plasma beam is slowed down in bulk from its initial drift velocity of 
$v_{s h}=0.4 c$ to about $0.15 c$, and further to almost a complete stop beyond $x / \Delta \gtrsim 60,000$. This is due to a strongly nonlinear backreaction of CRs on the ambient plasma particles. The bulk deceleration of the plasma drift starts at $t \approx 11 \gamma_{\max }^{-1}$, once the amplitude of magnetic-field turbulence becomes comparable to the strength of the homogeneous field, and the drift speed drops to $\sim 0.15 c$ at $t \approx 12.5 \gamma_{\max }^{-1}$ (compare Fig. 4 in [4]). Further growth of the turbulence is thus limited in this region and the instability saturates. This effect was already observed in studies with periodic numerical boxes and now it is re-confirmed here with the help of more realistic modeling.

Strong density and velocity fluctuations observed in the ambient plasma (e.g., the ion distribution in Fig. 3) lead to enhanced plasma heating through compression into filaments. This accounts for the irregular phase-space structure visible in Figure 4. A new effect, revealed through our modeling with open boundaries, can be observed at $x / \Delta \approx 35,000$, where the plasma beam, incoming with the initial drift velocity, collides with the slowed-down plasma. The magnetic modes are then compressed, additional plasma heating occurs, and a population of ions reflected from the compressed structure appears. The latter can lead to additional modifications of the turbulent structure due to, e.g., ion-beam streaming instabilities (e.g., [9]).

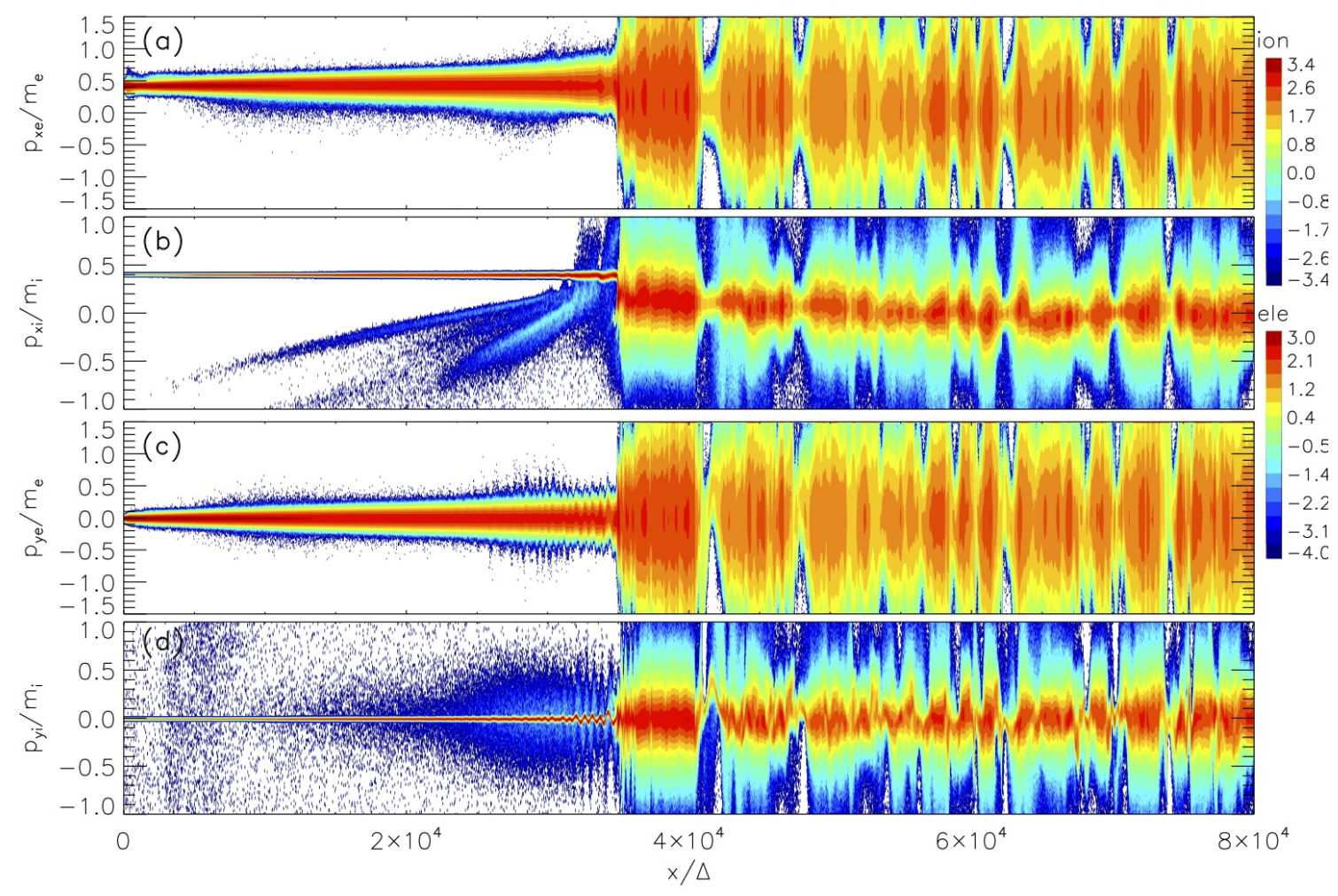

Figure 4: Phase-space distribution of plasma particles at the end $\left(t=20 \gamma_{\max }^{-1}\right)$ of our large-scale test run with the box width of $L_{x}=80,000 \Delta$. The upper two panels show $x$ - $p_{x}$ distributions of the $(a)$ beam electrons and $(b)$ the beam ions. The lower panels $\left(c\right.$ and $d$ ) show the corresponding distributions in $x-p_{y}$. The plasma at locations $x / \Delta>35,000$ is in a region of strong non-resonant turbulence that nonlinearly backreacts on $\mathrm{CR}$ and beam particles leading to their turbulent motion and heating. A prominent manifestation of this interaction is a slow-down of the beam drift. 


\section{Summary}

Magnetic-field generation through nonresonant cosmic-ray-current-driven instability operating at shocks of young supernova remnants has been studied here with PIC simulations that for the first time use a realistic setup with open boundaries, which accounts for mass conservation in decelerating flows. Our preliminary results demonstrate magnetic-field amplification as observed in earlier studies with periodic simulation boxes and re-confirm the expected effects of backreaction of CRs on the ambient plasma that manifest themselves by a decrease in the plasma drift velocity in the region of strong turbulence and significant turbulent motion and heating. The detail spatiotemporal structure of the shock precursor and the physics of the CR backreaction and magnetic field saturation processes are currently under investigation with large-scale numerical experiments.

\section{Acknowledgments}

This work used the HPC resources provided by The North-German Supercomputing Alliance (HLRN) and the Pleiades facility at the NASA Advanced Supercomputing (NAS). The work of J.N. is supported by Narodowe Centrum Nauki through research project DEC-2013/10/E/ST9/00662. M.P. acknowledges support through grants PO 1508/1-1 and PO 1508/1-2 of the Deutsche Forschungsgemeinschaft.

\section{References}

[1] Bell, A. R., 2004, MNRAS, 353, 550

[2] Niemiec, J., Pohl, M., Stroman, T., \& Nishikawa, K.-I., 2008, ApJ, 684, 1174

[3] Riquelme, M. A., \& Spitkovsky, A., 2009, ApJ, 694, 626

[4] Stroman, T., Pohl, M., \& Niemiec, J., 2009, ApJ, 706, 38

[5] Gargaté, L., Fonseca, R. A., Niemiec, J., Pohl, M., Bingham, R., \& Silva, L. O., 2010, ApJL, 711, L127

[6] Winske, D., Leroy, M.M., 1984, JGR, 89, 2673

[7] Luo, Q., Melrose, D., 2009, MNRAS, 397, 1402

[8] Ohira, Y., Reville, B., Kirk, J.G., \& Takahara, F., 2009, ApJ, 698, 445

[9] Niemiec, J., Pohl, M., Bret, A., \& Wieland, V., 2012, ApJ, 759, 73

[10] Greenwood, A. D., Cartwright, K. L., Luginslund, J. W., \& Baca, E.A., 2004, J. Comp. Phys., 201, 665

[11] Buneman, O., 1993, Computer Space Plasma Physics, ed. H. Matsumoto \& Y. Omura (Terra Scientific Publishing Company), 67-84 